\title{
Sonic hedgehog signaling in kidney fibrosis: a master communicator
}

\author{
Dong Zhou ${ }^{1}$, Roderick J. Tan ${ }^{2}$, \& Youhua Liu ${ }^{1,3 *}$ \\ ${ }^{1}$ Department of Pathology, University of Pittsburgh School of Medicine, Pittsburgh, Pennsylvania 15261, USA; \\ ${ }^{2}$ Department of Medicine, University of Pittsburgh School of Medicine, Pittsburgh, Pennsylvania 15261, USA; \\ ${ }^{3}$ State Key Laboratory of Organ Failure Research, National Clinical Research Center of Kidney Disease, Nanfang Hospital, Southern Medical \\ University, Guangzhou 510515, China
}

Received January 26, 2016; accepted March 6, 2016; published online June 21, 2016

\begin{abstract}
The hedgehog signaling cascade is an evolutionarily conserved pathway that regulates multiple aspects of embryonic development and plays a decisive role in tissue homeostasis. As the best studied member of three hedgehog ligands, sonic hedgehog (Shh) is known to be associated with kidney development and tissue repair after various insults. Recent studies uncover an intrinsic link between dysregulated Shh signaling and renal fibrogenesis. In various types of chronic kidney disease (CKD), Shh is upregulated specifically in renal tubular epithelium but targets interstitial fibroblasts, thereby mediating a dynamic epithelial-mesenchymal communication (EMC). Tubule-derived Shh acts as a growth factor for interstitial fibroblasts and controls a hierarchy of fibrosis-related genes, which lead to the excessive deposition of extracellular matrix in renal interstitium. In this review, we recapitulate the principle of Shh signaling, its activation and regulation in a variety of kidney diseases. We also discuss the potential mechanisms by which Shh promotes renal fibrosis and assess the efficacy of blocking this signaling in preclinical settings. Continuing these lines of investigations will provide novel opportunities for designing effective therapies to improve CKD prognosis in patients.
\end{abstract}

\section{Sonic hedgehog, Gli, tubular cells, fibroblast, renal fibrosis}

Citation: $\quad$ Zhou, D., Tan, R.J., and Liu, Y. (2016). Sonic hedgehog signaling in kidney fibrosis: a master communicator. Sci China Life Sci 59, 920-929. doi: 10.1007/s11427-016-0020-y

\section{INTRODUCTION}

It is estimated that the prevalence of chronic kidney disease (CKD) is about $10 \%-12 \%$ of the adult population worldwide (Coresh et al., 2007; Zhang et al., 2012; Zoccali et al., 2010). As a general rule, tissue fibrosis develops in virtually every form of CKD, as both a consequence and a characteristic feature of disease progression. From a cell biological standpoint, the intricate process of renal fibrosis has been linked, more or less, to an inappropriate activation of some key signaling pathways, such as transforming growth factor- $\beta$ (TGF- $\beta$ ), renin-angiotensin system (RAS), Wnt/ $\beta$ -

*Corresponding author (email: liuy@upmc.edu) catenin, and sonic hedgehog (Shh) (He and Dai, 2015; Meng et al., 2015; Santos et al., 2012; Sweetwyne et al., 2014; Tan et al., 2014). Most of the time, these diverse signals reciprocally crosstalk through cell-to-cell communication that involves all resident cells in the kidney, as well as the infiltrating cells (Duffield, 2014; Liu, 2011; Zeisberg and Neilson, 2010). In the past several years, the role and mechanisms of TGF- $\beta$, RAS and Wnt $/ \beta$-catenin signaling in the pathogenesis of CKD have been well documented (Meng et al., 2015; Sweetwyne et al., 2014; Tan et al., 2014; Zhou et al., 2015). Therefore, the scope of the present review is focused on our current understanding of Shh signaling in renal fibrogenesis.

Hedgehog signaling is an evolutionarily conserved, 
developmental pathway that plays an essential role in regulating mammalian embryonic development (Robbins et al., 2012). Hedgehog was identified in genetic screens in Drosophila in 1980, and the vertebrate hedgehog proteins were discovered in 1993 (Krauss et al., 1993; Nusslein-Volhard and Wieschaus, 1980). Since then, investigations on the role of hedgehog signaling in organ development and diseases progression have rapidly accelerated. There are three hedgehog ligands in mammals: Sonic hedgehog (Shh), Indian hedgehog (Ihh) and Desert hedgehog (Dhh), of which Shh is the best characterized (Lum and Beachy, 2004). Accumulating studies have demonstrated an activated Shh signaling in fibrotic CKD, suggesting a potential connection between aberrant regulation of this signaling and kidney fibrosis (Ding et al., 2012; Fabian et al., 2012; Kramann et al., 2015; Rauhauser et al., 2015; Zhou et al., 2014).

In this review, we will summarize the key features of Shh signaling and its regulation in various forms of CKD, and discuss the targets and the modes of action of Shh, as well as its relevant signal transduction routes. We will also provide the perspectives of several strategies to intervene Shh signaling for an effective therapy of the patients with fibrotic CKD.

\section{SONIC HEDGEHOG SIGNALING: COMPONENTS, ROUTES AND MECHANISM}

\section{Shh ligand}

As a morphogen, Shh is important in tissue patterning during embryonic development by controlling multiple biological processes including cell fate determination, cell proliferation and differentiation (Gill and Rosenblum, 2006; Pan et al., 2013). Human Shh is synthesized as a $45 \mathrm{kD}$ precursor protein with 462 amino acids, in which the first 23 amino acids serves as a signal peptide. It is autocatalytically cleaved to produce a $19 \mathrm{kD}$ amino-terminal fragment (N-Shh) and a $25 \mathrm{kD}$ carboxyl-terminal domain (C-Shh), and then secreted into the extracellular space. $\mathrm{N}$-Shh retains all known signaling capabilities, while C-Shh possesses protease activity.

During the cleavage, a cholesterol molecule is added to the carboxyl end of the N-terminal domain, which is involved in trafficking, secretion and receptor interaction of the Shh ligand (Figure 1A). Typically, secreted Shh contains two covalent modifications, a C-terminal cholesterol moiety and a palmitoyl group to the $\mathrm{N}$-terminal of the processed N-Shh. Despite its dual lipid modification and tight association with cell membranes, the Shh protein acts directly on distant cells in developing tissues. In vertebrates, this remote action requires the transmembrane transporter-like protein Dispatched (Disp) and Skinny hedgehog (Skn), which mediate the release of Shh from secreting cells (Lum and Beachy, 2004). Shh elicits its biological activity via both an autocrine and paracrine fashion.

\section{Canonical Shh signaling}

Shh transduces its signaling across the plasma membrane in the responding cells via either the canonical or noncanonical pathways. Canonical Shh signaling is the major pathway which has been studied intensively in vertebrate embryogenesis and tumorigenesis over the past few decades (Lum and Beachy, 2004). When secreted Shh ligand reaches its target cell, it binds with high affinity to the cell surface receptor, a twelve-pass transmembrane protein Patched-1 (Ptch1), to initiate the signal (Ingham, 2012). In the absence of Shh, Ptch1 normally represses the activity of Smoothened (Smo), the seven-pass transmembrane spanning receptor which is the major signaling component and switch in this pathway. The zinc-finger transcriptional factors of Glioma-associated oncogenes (Gli) family are inhibited by the cytoplasmic protein Suppressor of Fused ( $\mathrm{SuFu})$ (Tukachinsky et al., 2010). Conversely, in the presence of Shh binding to Ptch1, there is relief of the inhibition of Smo. Accumulated Smo receptor turns on a complex network leading to the regulation of three Gli transcriptional factors, the activators Gli1 and Gli2 and the repressor Gli3. Activated Gli accumulates in the nucleus and then controls the transcription of target genes, such as cyclin D1, cyclin E and N-Myc. Of note, besides acting as a transcriptional repressor mediated by a truncated fragment, Gli3 exists as a full length form which is able to activate transcription in specific systems (Dai et al., 2002).

In mammals, Ptch1 has a homologue Ptch2 that shares 54\% sequence identity (Carpenter et al., 1998). All three mammalian hedgehog ligands bind both Ptch1 and Ptch2 receptors with similar affinity, making it difficult to discriminate the receptor specificity. However, Ptch2 appears to have a distinct downstream signaling role from Ptch1, as it is expressed at much higher levels in specific organs such as testis and ovary (O'Hara et al., 2011). In the absence of ligand binding, Ptch2 has a decreased ability to inhibit the activity of Smo, compared with Ptch1 (Alfaro et al., 2014; Zhulyn et al., 2015). Structurally, Ptch protein has a sterol sensing domain (SSD), which is essential for inhibiting the activity of Smo (Lindstrom et al., 2006). Normally, Ptch acts as a sterol pump and removes oxysterols produced by 7-dehydrocholesterol reductase. Upon binding of Shh protein or a mutation in the SSD of Ptch, the pump is turned off, which allows oxysterols to accumulate around Smo. This accumulation of sterols renders Smo to become active or stay on the membrane for a longer period of time (Reichrath and Reichrath, 2013). In contrast to Drosophila, vertebrates possess another level of hedgehog regulation through ligand-dependent antagonism mediated by Hh-interacting protein 1 (Hhip1). Hhip and Smo colocalize at the cell surface, Hhip has no effect on activity of Smo but modulates Smo localization. In addition, Hhip1 also sequesters hedgehog ligands (Ochi et al., 2006; Zhao et al., 2014). 

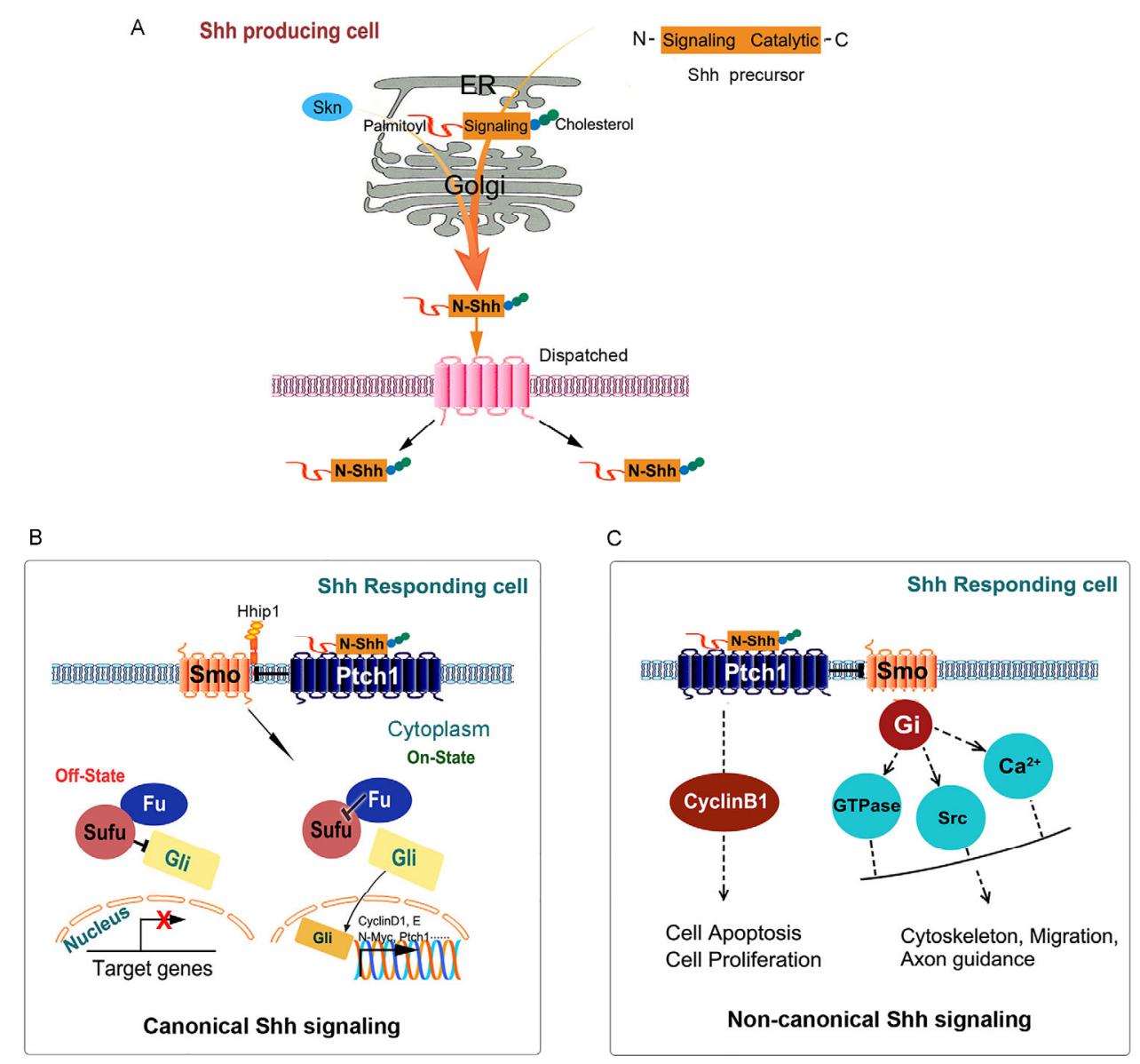

Figure 1 Schematic illustration of the Shh signaling pathway. A, In Shh-producing cells, the Shh precursor is proteolytically cleaved to generate the N-Shh in the endoplasmic reticulum (ER). Secreted Shh contains two covalent cholesterol modifications: a C-terminal cholesterol moiety and a palmitoyl group is added at the N-terminus. Skn and Dispatched, mediate the release of Shh ligand. B, The canonical Shh signaling. In the inactive state, the transmembrane protein receptor Ptch1 interacts with and inhibits the activity of a seven transmembrane protein, Smo. Interactions with cytoplasmic proteins, including Fused and Sufu, the transcription factors Gli are prevented from entering the nucleus and downstream target genes expression are repressed. In the active state, Shh binding to Ptch1, which allows Smo activation, thereby activating the cascade that leads to the Gli transcription factors to exert their effects in the nucleus. C, The non-canonical Shh signaling. There are two types of non-canonical Shh signaling pathways, one is Ptch1-dependent which regulates cell apoptosis and proliferation, the second is Smo-dependent which associate with modulation of actin cytoskeleton-dependent processes. Shh, Sonic hedgehog. N-Shh, N-terminal Sonic hedgehog. Skn, Skinny hedgehog. Ptch1, Patched-1. Smo, Smoothened. Hhip1, Hedgehog-interacting protein 1. Fu, Fused. Sufu, Suppressor of fused. Gli, Glioma-associated oncogenes.

\section{Non-canonical Shh signaling}

It is increasingly recognized that not all Shh signaling proceeds through Gli protein activation, thanks to the studies in the fields of cancer biology, neurological disorders and metabolic diseases (Aberger et al., 2012; Jenkins, 2009; Teperino et al., 2014; Zhao et al., 2010). The definition of "non-canonical Shh signaling" refers to the cellular responses to Shh ligand that are independent of the activation of the Gli family of transcription factors. At least two separate non-canonical Shh signaling pathways are characterized: one is through Ptch1 but is unrelated to its inhibition on Smo; another is through Smo but is beyond Gli regulation (Figure 1C) (Brennan et al., 2012).

In the non-canonical Shh signaling engaged by Ptch1, Ptch1 is shown to independently induce human embryonic kidney 293T cells apoptosis provoked by Shh deprivation
(Thibert et al., 2003). However, overexpression of Smo could not protect against cell death. Structurally, the C-terminal cytoplasmic domain of Ptch1 is a substrate for caspase-3, -7, and -8. Cleavage of Ptch1 is critical for apoptosis because mutation in the caspase site abolishes Ptch1-induced cell death. In addition, Ptch1 undergoes proteolytic processing at the C-terminus and the soluble C-terminal domain (CTD) translocates to the nucleus and mediates a new form of signal transduction (Kagawa et al., 2011). Different from Drosophila (Johnson et al., 2000), the CTD is not required for the canonical signal transduction but most likely has a distinct function in apoptosis and/or proliferation in mammals (Makino et al., 2001; Nieuwenhuis et al., 2007; Thibert et al., 2003). Besides apoptosis, exogenous Ptch1 in $293 \mathrm{~T}$ cells induces the redistribution of cyclin B1 from the nucleus to the cytoplasm, 
resulting in reduced cell proliferation. This effect could be disrupted in the presence of Shh.

Another non-canonical Shh signaling mainly controls endothelial cells cytoskeletal reorganization and fibroblast migration through activating small GTPases and regulating fluctuations of calcium ions upon stimulation of Shh (Chinchilla et al., 2010; El-Zaatari et al., 2010; Polizio et al., 2011). It has been demonstrated that none of the three hedgehog ligands are able to induce Gli-target genes in human umbilical vein (HUVeC) or human cardiac microvascular endothelial cells $(\mathrm{HMVeC})$, indicating that endothelial cells do not respond to hedgehog through the canonical pathway. However, all three hedgehog proteins promote endothelial cell tubulogenesis in 3D cultures in a Smodependent manner (Polizio et al., 2011). Consistent with the required cytoskeletal rearrangement for tubulogenesis, Shh, Ihh and Dhh all stimulate the small GTPase RhoA and actin stress fibers formation (Chinchilla et al., 2010). This effect, mediated by Smo and $G$ proteins, defines a new noncanonical hedgehog pathway. In this system, stimulation by hedgehog ligands results in the formation of actin stress fibers within minutes, thus suggesting a non-canonical pathway based on the time course and the lack of detectable Gli-dependent transcription. Reminiscent of cytoskeleton remodeling, Shh could activate non-canonical signaling pathway to control axon guidance by stimulating the activity of Src family kinase (Yam et al., 2009).

\section{SHH SIGNALING AND CKD}

\section{Shh induction and localization in CKD}

Shh is known to be expressed in epithelial cells during development of several organs including kidney. In adult kidneys, however, Shh expression is largely silenced. Re-activation of Shh signaling has been implicated in the pathogenesis of tissue fibrosis in many organs including kidney, lung and liver (Chung et al., 2016; Cigna et al., 2012; Zhou et al., 2014).

As the major constituent of renal parenchyma, kidney tubular epithelium is the epicenter of various toxic, ischemic, metabolic and immunologic injuries (Li et al., 2012; Zhou et al., 2012). Although the damaged tubular cells may undergo a variety of maladaptive changes such as partial epithelial-mesenchymal transition (EMT), cell cycle arrest, defects in cell metabolism (Grande et al., 2015; Kang et al., 2015; Liu, 2010; Lovisa et al., 2015), one common consequence of these diverse responses is converting to a secretory phenotype (Zhou and Liu, 2016). Indeed, marked induction of Shh protein is observed in the fibrotic kidneys in all commonly used CKD models, including folic acid (FA), unilateral ureteral obstruction (UUO), ischemia reperfusion injury (IRI), adriamycin (ADR) and 5/6 nephrectomy, although it is barely detectable in normal kidneys (Ding et al., 2012; Fabian et al., 2012; Rauhauser et al.,
2015; Zhou et al., 2014). In human biopsy specimens, Shh is also specifically induced in renal tubular epithelium of the diseased kidney, regardless of the initial etiologies (Zhou et al., 2014). Meanwhile, the sensor receptor Ptch1 and transcriptional factor Gli1, Gli2 are also upregulated in high-grade fibrotic human kidneys (Kramann et al., 2015; Zhou et al., 2014). These results suggest that Shh upregulation is a common pathologic finding in a wide variety of kidney diseases.

Shh induction is predominantly localized in renal tubular epithelium (Figure 2), whereas interstitial cells in the expanded interstitium are largely negative for Shh staining. Most importantly, upregulation of Shh protein is an extremely early event in different models in vivo, suggesting a potential role for Shh in triggering the first wave of defense mechanism after tissue damage. In vitro, a variety of pathologic cues such as TGF- $\beta 1$ and Wnt induces Shh mRNA expression and protein secretion by tubular epithelial cells. Based on these observations, we recently propose that Shh is a novel, inducible, tubule-derived growth factor that mediates epithelial-mesenchymal communication (EMC) after kidney injury (Ding et al., 2012; Zhou et al., 2014).

\section{Shh promotes kidney fibrosis}

The functionality of Shh in the development and progression of CKD has been extensively studied by both gain- and loss-of function approaches. Emerging evidence indicates that along with the progression of kidney injuries, hyperactive Shh signaling causes fibroblast activation, proliferation and matrix over-production by multiple mechanisms, primarily via a mode of epithelial-mesenchymal interaction.

In mouse model of IRI, overexpression of Shh transgene through a hydrodynamic-based gene transfer approach promoted fibroblast proliferation and matrix overproduction and deposition, ultimately leading to an increased kidney fibrosis and aggravated kidney dysfunction (Zhou et al., 2014). The population of Shh-responsive Gli1-positive cells is markedly expanded in renal interstitium after Shh transgene expression. In vitro, human recombinant Shh protein activated Ptch1 and Gli1 and induced $\alpha$-smooth muscle actin ( $\alpha$-SMA), desmin, Snail1, fibronectin, and collagen I expression (Ding et al., 2012; Zhou et al., 2014), suggesting a critical role for Shh signaling in regulating myofibroblast activation and matrix production. Shh also promotes cultured rat kidney fibroblast proliferation and stimulates the induction of numerous proliferation-related genes (Zhou et al., 2014). Shh ligand also triggers pericytelike cell proliferation in vitro (Fabian et al., 2012), suggesting a role for this pathway in regulating cell cycle progression of myofibroblast progenitors during the development of renal fibrosis.

Using conditional knockout mouse model, we recently show that tubule-specific deletion of Shh reduces fibrotic lesions after kidney injury (data not shown), suggesting that Shh not only is sufficient but also necessary for fibrosis 

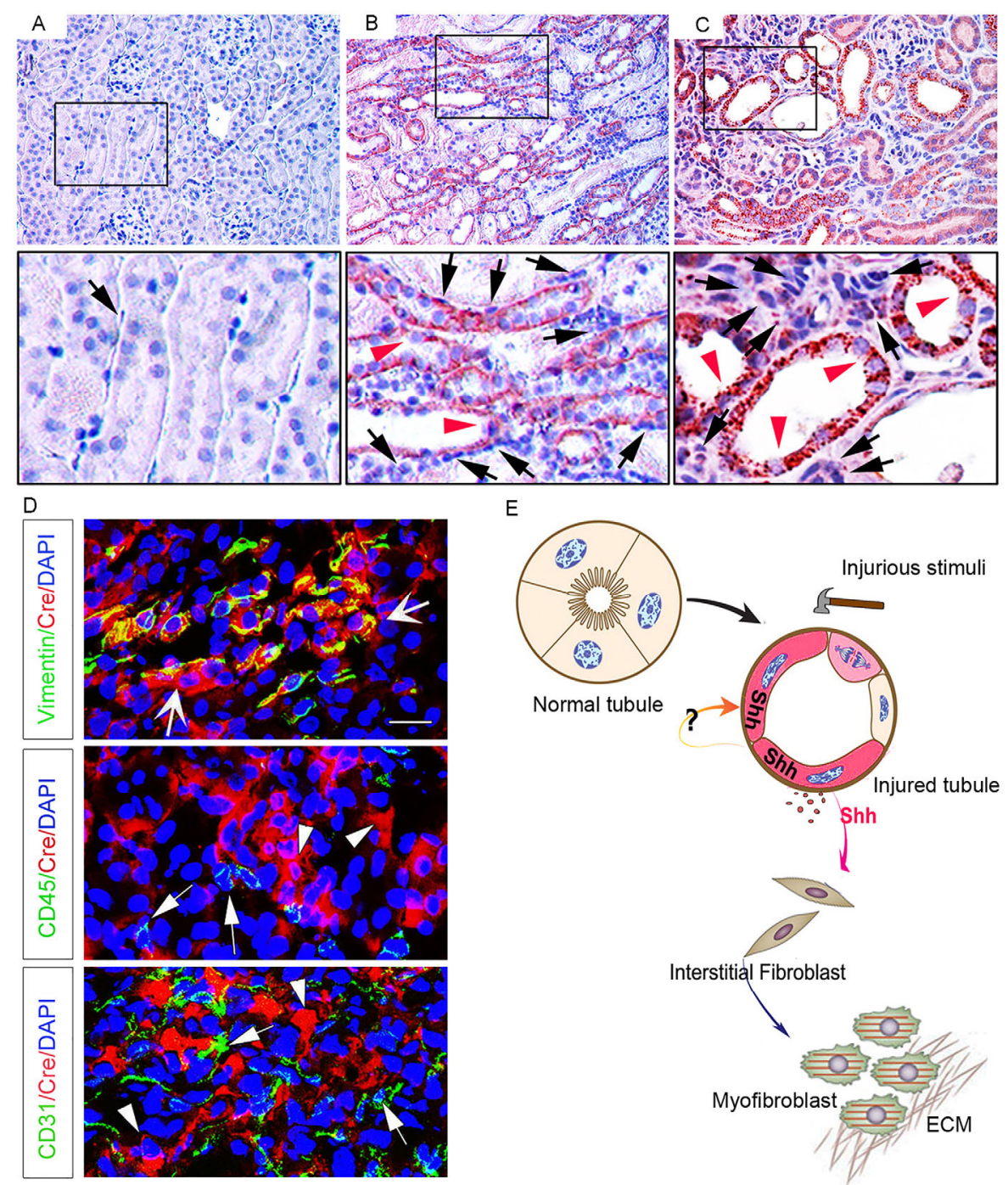

Figure 2 Tubule-derived Shh targets interstitial fibroblasts. A-C, Representative micrographs of immunohistochemical staining show marked induction of Shh protein after renal ischemia reperfusion injury (IRI) at 1 (B) and 10 days (C), respectively. Shh is barely detectable in sham control (A). At 1 day after IRI, Shh expression was significantly induced (B, red arrowhead) in renal tubules. At 10 days, sustained activation of Shh in renal tubules was observed (C, red arrowhead). D, Identification of the interstitial fibroblasts as Shh-responsive cells in fibrotic kidneys. Transgenic Gli1-CreER ${ }^{\mathrm{T2}}$ mice were subjected to IRI for 10 days, and kidneys subjected to double immunostaining for Cre recombinase (red) and various cell type-specific markers (green). Wide arrows indicate cells with positive staining for both vimentin and Cre; arrowheads denote Cre-positive cells; arrows show CD45- or CD31-positive cells. Panel D was reproduced from our published paper (Zhou et al., 2014) with permission. E, Schematic diagram shows that Shh mediates epithelial-mesenchymal communication between injured tubules and interstitial fibroblasts, leading to fibroblast activation and proliferation, as well as matrix overproduction.

development in the kidney. This conclusion is also supported by genetic and pharmacologic manipulations of the Shh downstream mediators Smo or Gli proteins. It is shown that the kidneys in Gli1-deficient mice are protected against the development of tubulointerstitial fibrosis after UUO (Ding et al., 2012; Rauhauser et al., 2015). Smo inhibitors such as cyclopamine also repress the induction of Gli1, Snail1 and $\alpha$-SMA, and reduce matrix expression and mitigate fibrotic lesions in obstructive nephropathy (Ding et al., 2012; Rauhauser et al., 2015), although it does not affect renal Shh expression. Another study demonstrates that Gli2 in Shh pathway is expressed in myofibroblast progenitors, and drive cell-cycle progression in cultured mesenchymal stem cell-like progenitors (Kramann et al., 2015). Myofibroblast-specific deletion of Gli2 or overexpression of Gli3 repressor attenuates renal fibrosis after UUO (Kramann et al., 2015). In addition, suppression of Shh signaling is also associated with decreased macrophage infiltration after obstructive injury (Rauhauser et al., 2015).

\section{TARGETS AND MECHANISM OF SHH SIGNALING IN KIDNEY FIBROSIS}

\section{Fibroblasts as a major target of canonical Shh signaling}

Given that Shh is a secreted protein mainly produced by renal tubular epithelium, an important question that needs to 
be clarified is the cellular targets of Shh in diseased kidney. To address this issue, three independent groups utilized the Gli1 ${ }^{\text {lacZ }}$ mutant mice, the so-called hedgehog-reporter mice, which harbor a $\beta$-galactosidase $(\beta-\mathrm{Gal})$ "knock-in" mutation. Under the control of the native Gli1 upstream promoter/enhancer elements, lacZ expression in these mice authentically recapitulates the expression pattern of the endogenous Gli1 mRNA. Results show that interstitial fibroblasts, including platelet-derived growth factor receptor (PDGFR)$\beta$-positive pericytes, are identified as principal Shhresponding cells in vivo in UUO and IRI models (Ding et al., 2012; Fabian et al., 2012; Rauhauser et al., 2015; Zhou et al., 2014). Further studies using Gli1-Cre mice have demonstrated that the Shh-responding cell population does not include CD31-positive endothelial cells and CD45positive monocytes in renal interstitium (Zhou et al., 2014) (Figure 2D). These findings are consistent with the observations that cultured kidney fibroblasts respond to Shh stimulation in vitro, leading to an enhanced cell proliferation and myofibroblastic activation. Intriguingly, Shh had no effect on the proliferation of renal tubular epithelial cells (Zhou et al., 2014). Therefore, as a tubule-derived growth factor, Shh specifically targets interstitial fibroblasts and mediates epithelial-mesenchymal communication via a paracrine fashion (Figure 2).

It should be pointed out that Gli1 expression only reflects the activation of the canonical hedgehog signaling in the responding cells. In this regard, possibility exists that other types of cells in the kidney besides interstitial fibroblasts, such as tubular epithelial cells, endothelial cells and infiltrated inflammatory cells, may also respond to Shh via Gli-independent, non-canonical pathway. More studies are warranted in this area to define the role and mechanism of Shh in regulating the activities of many other kidney cells under pathologic conditions.

\section{Crosstalk with other signaling pathways in kidney fibrosis}

Increasing evidence suggests that Shh signal interacts with several key signaling pathways and operates in a coordinated fashion during renal fibrogenesis. For instance, besides Shh signaling pathway, TGF- $\beta 1$, Wnt/ $\beta$-catenin and Notch pathways are well recognized to play crucial roles in the evolution of renal fibrosis in CKD (Bielesz et al., 2010; He and Dai, 2015; He et al., 2009; Tan et al., 2014; Zhou et al.,
2016). However, the role of Shh signaling is quite unique in this process in that it specifically targets interstitial cells, while it is mainly produced by the injured tubules. In this way, Shh acts as a master communicator between the injured tubular epithelial cells and interstitial fibroblasts, leading to fibroblast activation and expansion, which plays a central role in renal fibrogenesis (Fabian et al., 2012; Kramann et al., 2015; Zhou et al., 2014). In contrast, the major targets of Wnt and Notch signals are tubular epithelial cells, which then indirectly promote kidney fibrosis through the processes such as partial EMT (Liu, 2006, 2010).

Over the past several years, evidence of crosstalk among Shh, Wnt, Notch, TGF- $\beta 1$ and other signaling pathways has been frequently reported in renal fibrosis. For example, Shh is known to upregulate $\mathrm{Wnt} 2 \mathrm{~b}$ and Wnt5a (Katoh and Katoh, 2009), as well as Notch ligand Jaggad2 (Katoh and Katoh, 2008). Wnts could induce Shh expression in epithelial cells, and both Wnt and Shh signaling can induce Snail1 expression in fibrotic kidneys (Ding et al., 2012; Wang et al., 2011; Zhou and Liu, 2015). Gli1 could induce Snaill and promote $\beta$-catenin nuclear translocation in epithelial cells (Li et al., 2007). TGF- $\beta 1$ is reported to induce several key components of the Wnt, Shh and Notch signaling pathways (Bielesz et al., 2010; Wang et al., 2011). Therefore, crosstalk between these signal pathways has the potential to orchestrate complex cellular responses to extracellular stimuli. These findings present new challenges-as well as opportunities-for discovering novel interventional strategies.

\section{STRATEGIES TO TARGET SHH SIGNALING IN KIDNEY FIBROSIS}

In view of the central role of Shh signaling in renal fibrosis, targeting this pathway might be an effective strategy to halt and even to reverse the progression of CKD. In theory, the Shh signaling could be blocked at different levels along the signal transduction route. Recently published literature describes small molecule inhibitors in Shh pathway as the following categories (Gonnissen et al., 2015; Ruat, 2015): inhibitors targeting Smo; strategies to target the upstream $\mathrm{N}-\mathrm{Shh}$ ligand; and inhibitors targeting the downstream transcription factors Gli1 and Gli2 (Figure 3). The transla tion of these exciting new pharmacologic therapies to the treatment of kidney diseases has just begun in the preclinical setting (Table 1). In this review, we will focus on the

Table 1 The strategies to target Shh signaling in fibrotic CKD ${ }^{\text {a) }}$

\begin{tabular}{|c|c|c|c|c|c|}
\hline Disease model & producing-cells & Responding cells & Target/inhibitor & Outcome & References \\
\hline UUO & Tubular cells & Fibroblast & Smo/cyclopamine & Improved & (Ding et al., 2012) \\
\hline IRI & Tubular cells & Fibroblast & Smo/cyclopamine & Improved & (Zhou et al., 2014) \\
\hline UUO & Tubular cells & Pericyte/fibroblast & Smo/IPI-926 & Negative & (Fabian et al., 2012) \\
\hline UUO & Tubular cells & & Gli2/darinaparsin & Improved & (Kramann et al., 2015) \\
\hline UUO & Tubular cells & Pericyte/macrophage & Smo/cyclopamine & Improved & (Rauhauser et al., 2015) \\
\hline
\end{tabular}

a) UUO, unilateral ureteral obstruction. IRI, ischemia reperfusion injury 


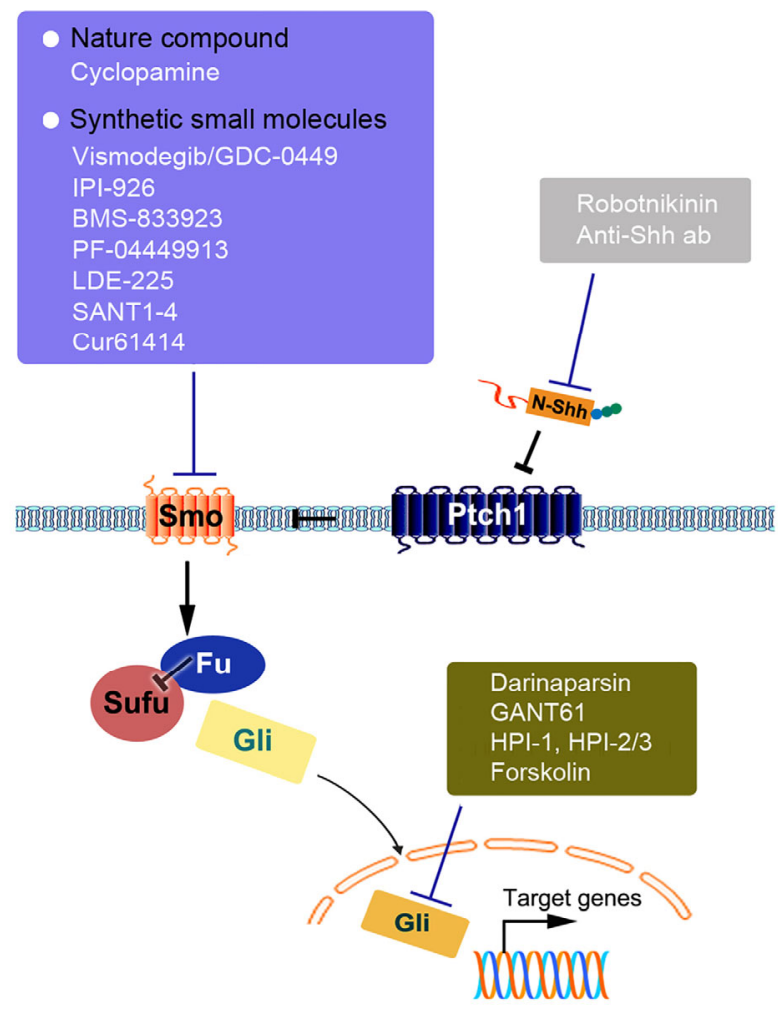

Figure 3 Therapeutic strategies to target Shh signaling pathway. The Shh signaling could be blocked at different levels, including Shh ligand inhibitors, Smo receptor inhibitors and transcriptional factors Gli inhibitors. Among these antagonists, Smo inhibitors (cyclopamine and IPI-926) and Gli inhibitors (Darinaparsin and GANT61) are tested in the setting of fibrotic CKD. Except for IPI-926 (Fabian et al., 2012), all Shh signaling inhibitors are able to attenuate renal fibrosis in experimental models.

Smo inhibitors and Gli inhibitors, which have been recently assessed for their efficacy in the treatment of kidney diseases.

\section{Smo inhibitors}

The most common method to target Shh pathway is through manipulation of Smo activity because it is the main signal transducer of this pathway. Smo is responsible for a large variety of developmental processes. It belongs to class $\mathrm{F}$ (Frizzled family) of the G-protein-coupled receptor (GPCR) superfamily (Ruiz-Gomez et al., 2007). Recently, robust clinical investigations implicate Smo as a novel therapeutic target in human cancers.

As the first member of a class of small molecular compounds that specifically inhibit the activity of Smo, cyclopamine (CPN) has been utilized in the treatment of experimental renal fibrosis (Ding et al., 2012; Rauhauser et al., 2015). CPN is a natural alkaloid derivative isolated from a plant of the corn lily family (Incardona et al., 1998; Strand et al., 2011). In renal fibrogenesis, targeting Smo with CPN inhibits fibroblast activation and matrix production in vitro, and it reduces matrix expression and mitigates fibrotic lesions in vivo after UUO and IRI (Ding et al., 2012; Zhou et al., 2014). Meanwhile, induction of the downstream target of Smo, Gli1 and Snail1, are largely abolished by CPN (Ding et al., 2012; Zhou et al., 2014). However, it does not affect upstream Shh ligand expression. The shortcomings of CPN are its short half-life and off-target effects at higher doses (Lipinski et al., 2008; Zhao et al., 2009). Therefore, a more soluble and potent CPN derivative, IPI-926 (Saridegib), has been explored in clinical trials for basal cell carcinoma (BCC) and metastatic pancreatic cancer. After UUO, IPI-926 abolishes Gli1 induction, but not Gli2 in vivo, suggesting the existence of Smo-independent Gli activation in the model. In contrast to CPN, IPI-926 did not attenuate renal fibrosis (Fabian et al., 2012). These differences might be due to the chemical diversity of Smo modulators and their different pharmacological properties, because there are at least two well-characterized ligandbinding pockets presented in Smo receptor: one in the extracellular domain (ECD), and one in the TM domain.

The discovery of CPN and its derivative has heralded extensive research for development of Smo antagonist. A major breakthrough is the recent approval of a novel Smo antagonist Erivedge/Vismodegib (GDC-0449) in the Shh field by the FDA for treating metastatic BCC and locally advanced BCC deemed untreatable by surgery or radiation (Robarge et al., 2009). However, its effects in kidney diseases remain to be investigated and validated in the preclinical setting before considering any clinic trials.

\section{Gli inhibitors}

Although Smo has been considered as the most common target of Shh signaling, the importance of the downstream regulators of Smo, such as $\mathrm{SuFu}$ and Gli1/2 cannot be ignored. After all, Smo antagonists do not repress upstream ligands expression, whereas a compound directed against the downstream targets would be able to more effectively inhibit the entire pathway. In this regard, Darinaparsin, a novel organic arsenical with optimized pharmacokinetic properties, is currently in clinical studies for hematologic malignancies and solid tumors. As the antagonist of Gli proteins, Darinaparsin directly targets Gli2 protein and induces Gli-dependent cell-cycle arrest in renal myofibroblasts, thereby preventing myofibroblast proliferation and ameliorate kidney fibrosis in vivo (Kramann et al., 2015). Besides Darinaparsin, GANT61, a small-molecule inhibitor of Gli, also displayed the ability of ameliorating renal fibrosis in mice, even it is administered after injury (Kramann et al., 2015).

\section{SHH SIGNALING AND THE FIBROTIC DISEASE OF OTHER ORGANS}

Besides kidney, increasing evidence suggests that activation of Shh signaling is also associated with tissue fibrosis in other organs including lung and liver. In vitro, it has been 
well documented that recombinant Shh protein induces an activated phenotype of cultured lung fibroblasts and hepatic stellate cells, as manifested by an augmented cell proliferation, migration, excessive ECM production (Hu et al., 2015; Yang et al., 2008). Shh signaling is upregulated in animal models and patients with idiopathic pulmonary fibrosis (IPF), other interstitial lung diseases and nonalcoholic fatty liver disease (NAFLD), with nuclear accumulation of Gli1, Gli2 in the fibrotic areas and an increased expression of the Shh downstream target genes. In lung, Shh is predominantly expressed in type II like epithelial cells of terminal bronchioles and alveoli (Bolanos et al., 2012). Shh activation causes aberrant epithelium-fibroblast interactions and directly triggers pulmonary fibrosis after injury ( $\mathrm{Hu}$ et al., 2015; McGowan and McCoy, 2013). Unlike lung, Shh is found in both ballooned hepatocyte and hepatic stellate cells in the liver of NAFLD, which eventually leads to liver fibrosis or cirrhosis (Hirsova and Gores, 2015; Rangwala et al., 2011). By using hydrodynamics-based gene transfer approach, hepatic expression of Shh induces liver fibrosis, which is accompanied by concurrent activation of hepatic stellate cells, and upregulation of various fibrogenic genes (Chung et al., 2016). Meanwhile, hepatic stellate cells could produce Shh through an autocrine fashion to accelerate progression of liver diseases. In addition, activation of Shh signaling is also responsible for dermal fibrosis in systemic sclerosis (Horn et al., 2012). Therefore, activation of Shh signaling perhaps is a generalized mechanism leading to tissue fibrosis after injury.

\section{SUMMARY}

As a potent morphogen, Shh controls cell proliferation, differentiation and morphogenesis in embryonic kidney ( $\mathrm{Yu}$ et al., 2002). In the past few years, increasing evidence indicates that re-activation of Shh signaling plays a critical role in the onset and progression of kidney fibrosis after various insults. Shh protein is produced and secreted by the injured tubules but specifically targets interstitial fibroblasts, leading to their activation, proliferation and expansion. Through such a paracrine fashion, Shh acts as a master communicator and mediates epithelial-mesenchymal communication (EMC) during renal fibrogenesis. By characterizing the receptors, downstream regulators and transcriptional effectors in the Shh signaling, pharmacologic manipulation of this pathway is now being attempted in a variety of animal models of CKD in preclinical setting.

Our knowledge of Shh signaling remains in its infancy in the field of nephrology. Continuous exploration of renal pathogenesis linked with the Shh pathway and validation of pharmacological intervention strategies is an urgent task for researchers and nephrologists. There are many questions to be answered. For instance, since upregulation of Shh is an early event after injuries, what is the function of Shh in the setting of acute kidney injury? What is the role of Shh in glomerular diseases where the specialized epithelium (podocytes) communicates with mesangial cells? What is the extent of interaction between Shh and other pathologic signal pathways? It will be of great interest to see these different aspects of Shh signaling unveiled in the near future.

Compliance and ethics The author(s) declare that they have no conflict of interest.

Acknowledgements This work was supported by the National Natural Science Foundation of China (81130011, 81370839, 81521003), Guangdong Science Foundation (2014A030312014), Guangzhou Projects Grant (15020025), American Heart Association FTF (16990086), and National Institutes of Health Grants (DK064005, DK091239, DK106049).

Aberger, F., Kern, D., Greil, R., and Hartmann, T.N. (2012). Canonical and noncanonical Hedgehog/GLI signaling in hematological malignancies. Vitam Horm 88, 25-54.

Alfaro, A.C., Roberts, B., Kwong, L., Bijlsma, M.F., and Roelink, H. (2014). Ptch 2 mediates the Shh response in Ptch1 $/-$ cells. Development 141, 3331-3339.

Bielesz, B., Sirin, Y., Si, H., Niranjan, T., Gruenwald, A., Ahn, S., Kato, H., Pullman, J., Gessler, M., Haase, V.H., and Susztak, K. (2010). Epithelial Notch signaling regulates interstitial fibrosis development in the kidneys of mice and humans. J Clin Invest 120, 4040-4054.

Bolanos, A.L., Milla, C.M., Lira, J.C., Ramirez, R., Checa, M., Barrera, L., Garcia-Alvarez, J., Carbajal, V., Becerril, C., Gaxiola, M., Pardo, A., and Selman, M. (2012). Role of Sonic Hedgehog in idiopathic pulmonary fibrosis. Am J Physiol Lung Cell Mol Physiol 303, L978-990.

Brennan, D., Chen, X., Cheng, L., Mahoney, M., and Riobo, N.A. (2012). Noncanonical Hedgehog signaling. Vitam Horm 88, 55-72.

Carpenter, D., Stone, D.M., Brush, J., Ryan, A., Armanini, M., Frantz, G., Rosenthal, A., and de Sauvage, F.J. (1998). Characterization of two patched receptors for the vertebrate hedgehog protein family. Proc Natl Acad Sci USA 95, 13630-13634.

Chinchilla, P., Xiao, L., Kazanietz, M.G., and Riobo, N.A. (2010). Hedgehog proteins activate pro-angiogenic responses in endothelial cells through non-canonical signaling pathways. Cell Cycle 9, 570-579.

Chung, S.I., Moon, H., Ju, H.L., Cho, K.J., Kim, do Y., Han, K.H., Eun, J.W., Nam, S.W., Ribback, S., Dombrowski, F., Calvisi, D.F., and Ro, S.W. (2016). Hepatic expression of Sonic Hedgehog induces liver fibrosis and promotes hepatocarcinogenesis in a transgenic mouse model. J Hepatol 64, 618-627.

Cigna, N., Farrokhi, Moshai, E., Brayer, S., Marchal-Somme, J., Wemeau-Stervinou, L., Fabre, A., Mal, H., Leseche, G., Dehoux, M., Soler, P., Crestani, B., and Mailleux, A.A. (2012). The hedgehog system machinery controls transforming growth factor-beta-dependent myofibroblastic differentiation in humans: involvement in idiopathic pulmonary fibrosis. Am J Pathol 181, 2126-2137.

Coresh, J., Selvin, E., Stevens, L.A., Manzi, J., Kusek, J.W., Eggers, P., Van Lente, F., and Levey, A.S. (2007). Prevalence of chronic kidney disease in the United States. JAMA 298, 2038-2047.

Dai, P., Shinagawa, T., Nomura, T., Harada, J., Kaul, S.C., Wadhwa, R., Khan, M.M., Akimaru, H., Sasaki, H., Colmenares, C., and Ishii, S. (2002). Ski is involved in transcriptional regulation by the repressor and full-length forms of Gli3. Genes Dev 16, 2843-2848.

Ding, H., Zhou, D., Hao, S., Zhou, L., He, W., Nie, J., Hou, F.F., and Liu, Y. (2012). Sonic hedgehog signaling mediates epithelial-mesenchymal communication and promotes renal fibrosis. J Am Soc Nephrol 23, 801-813.

Duffield, J.S. (2014). Cellular and molecular mechanisms in kidney fibrosis. J Clin Invest 124, 2299-2306.

El-Zaatari, M., Zavros, Y., Tessier, A., Waghray, M., Lentz, S., Gumucio, D., Todisco, A., and Merchant, J.L. (2010). Intracellular calcium release and protein kinase $\mathrm{C}$ activation stimulate sonic hedgehog gene 
expression during gastric acid secretion. Gastroenterology 139, 2061-2071 e2062.

Fabian, S.L., Penchev, R.R., St-Jacques, B., Rao, A.N., Sipila, P., West, K.A., McMahon, A.P., and Humphreys, B.D. (2012). Hedgehog-Gli pathway activation during kidney fibrosis. Am J Pathol 180, 1441-1453.

Gill, P.S., and Rosenblum, N.D. (2006). Control of murine kidney development by sonic hedgehog and its GLI effectors. Cell Cycle 5, 1426-1430.

Gonnissen, A., Isebaert, S., and Haustermans, K. (2015). Targeting the Hedgehog signaling pathway in cancer: beyond Smoothened. Oncotarget 6, 13899-13913.

Grande, M.T., Sanchez-Laorden, B., Lopez-Blau, C., De Frutos, C.A., Boutet, A., Arevalo, M., Rowe, R.G., Weiss, S.J., Lopez-Novoa, J.M., and Nieto, M.A. (2015). Snaill-induced partial epithelial-tomesenchymal transition drives renal fibrosis in mice and can be targeted to reverse established disease. Nat Med 21, 989-997.

He, W., and Dai, C. (2015). Key fibrogenic signaling. Curr Pathobiol Rep 3, 183-192.

He, W., Dai, C., Li, Y., Zeng, G., Monga, S.P., and Liu, Y. (2009). Wnt/beta-catenin signaling promotes renal interstitial fibrosis. J Am Soc Nephrol 20, 765-776.

Hirsova, P., and Gores, G.J. (2015). Ballooned hepatocytes, undead cells, sonic hedgehog, and vitamin E: therapeutic implications for nonalcoholic steatohepatitis. Hepatology 61, 15-17.

Horn, A., Palumbo, K., Cordazzo, C., Dees, C., Akhmetshina, A., Tomcik, M., Zerr P., Avouac, J., Gusinde, J., Zwerina, J., Roudaut, H., Traiffort, E., Ruat, M., Distler, O., Schett, G., and Distler, J.H. (2012). Hedgehog signaling controls fibroblast activation and tissue fibrosis in systemic sclerosis. Arthritis Rheum 64, 2724-2733.

Hu, B., Liu, J., Wu, Z., Liu, T., Ullenbruch, M.R., Ding, L., Henke, C.A., Bitterman, P.B., and Phan, S.H. (2015). Reemergence of hedgehog mediates epithelial-mesenchymal crosstalk in pulmonary fibrosis. Am J Respir Cell Mol Biol 52, 418-428.

Incardona, J.P., Gaffield, W., Kapur, R.P., and Roelink, H. (1998). The teratogenic Veratrum alkaloid cyclopamine inhibits sonic hedgehog signal transduction. Development 125, 3553-3562.

Ingham, P.W. (2012). Hedgehog signaling. Cold Spring Harb Perspect Biol 4, a011221.

Jenkins, D. (2009). Hedgehog signalling: emerging evidence for non-canonical pathways. Cell Signal 21, 1023-1034.

Johnson, R.L., Milenkovic, L., and Scott, M.P. (2000). In vivo functions of the patched protein: requirement of the $\mathrm{C}$ terminus for target gene inactivation but not Hedgehog sequestration. Mol Cell 6, 467-478.

Kagawa, H., Shino, Y., Kobayashi, D., Demizu, S., Shimada, M., Ariga, H., and Kawahara, H. (2011). A novel signaling pathway mediated by the nuclear targeting of C-terminal fragments of mammalian Patched 1. PLoS One 6, e18638.

Kang, H.M., Ahn, S.H., Choi, P., Ko, Y.A., Han, S.H., Chinga, F., Park, A.S., Tao, J., Sharma, K., Pullman, J., Bottinger, E.P., Goldberg, I.J., and Susztak, K. (2015). Defective fatty acid oxidation in renal tubular epithelial cells has a key role in kidney fibrosis development. Nat Med 21, 37-46.

Katoh, Y., and Katoh, M. (2008). Hedgehog signaling, epithelial-tomesenchymal transition and miRNA. Int J Mol Med 22, 271-275.

Katoh, Y., and Katoh, M. (2009). Hedgehog target genes: mechanisms of carcinogenesis induced by aberrant hedgehog signaling activation. Curr Mol Med 9, 873-886.

Kramann, R., Fleig, S.V., Schneider, R.K., Fabian, S.L., DiRocco, D.P., Maarouf, O., Wongboonsin, J., Ikeda, Y., Heckl, D., Chang, S.L., Rennke, H.G., Waikar, S.S., and Humphreys, B.D. (2015). Pharmacological GLI2 inhibition prevents myofibroblast cell-cycle progression and reduces kidney fibrosis. J Clin Invest 125, 2935-2951.

Kramann, R., Schneider, R.K., DiRocco, D.P., Machado, F., Fleig, S., Bondzie, P.A., Henderson, J.M., Ebert, B.L., and Humphreys, B.D. (2015). Perivascular $\mathrm{Gli1}^{+}$progenitors are key contributors to injury-induced organ fibrosis. Cell Stem Cell 16, 51-66.

Krauss, S., Concordet, J.P., and Ingham, P.W. (1993). A functionally conserved homolog of the Drosophila segment polarity gene hh is ex- pressed in tissues with polarizing activity in zebrafish embryos. Cell 75, 1431-1444.

Li, X., Deng, W., Lobo-Ruppert, S.M., and Ruppert, J.M. (2007). Gli1 acts through Snail and E-cadherin to promote nuclear signaling by beta-catenin. Oncogene 26, 4489-4498.

Li, Y., Wen, X., and Liu, Y. (2012). Tubular cell dedifferentiation and peritubular inflammation are coupled by the transcription regulator Id1 in renal fibrogenesis. Kidney Int 81, 880-891.

Lindstrom, E., Shimokawa, T., Toftgard, R., and Zaphiropoulos, P.G. (2006). PTCH mutations: distribution and analyses. Hum Mutat 27, 215-219.

Lipinski, R.J., Hutson, P.R., Hannam, P.W., Nydza, R.J., Washington, I.M., Moore, R.W., Girdaukas, G.G., Peterson, R.E., and Bushman, W. (2008). Dose- and route-dependent teratogenicity, toxicity, and pharmacokinetic profiles of the hedgehog signaling antagonist cyclopamine in the mouse. Toxicol Sci 104, 189-197.

Liu, Y. (2006). Renal fibrosis: new insights into the pathogenesis and therapeutics. Kidney Int 69, 213-217.

Liu, Y. (2010). New insights into epithelial-mesenchymal transition in kidney fibrosis. J Am Soc Nephrol 21, 212-222.

Liu, Y. (2011). Cellular and molecular mechanisms of renal fibrosis. Nat Rev Nephrol 7, 684-696.

Lovisa, S., LeBleu, V.S., Tampe, B., Sugimoto, H., Vadnagara, K., Carstens, J.L., Wu, C.C., Hagos, Y., Burckhardt, B.C., Pentcheva-Hoang, T., Nischal, H., Allison, J.P., Zeisberg, M., and Kalluri, R. (2015). Epithelial-to-mesenchymal transition induces cell cycle arrest and parenchymal damage in renal fibrosis. Nat Med 21, 998-1009.

Lum, L., and Beachy, P.A. (2004). The Hedgehog response network: sensors, switches, and routers. Science 304, 1755-1759.

Makino, S., Masuya, H., Ishijima, J., Yada, Y., and Shiroishi, T. (2001). A spontaneous mouse mutation, mesenchymal dysplasia (mes), is caused by a deletion of the most C-terminal cytoplasmic domain of patched (ptc). Dev Biol 239, 95-106.

McGowan, S.E., and McCoy, D.M. (2013). Platelet-derived growth factor-A and sonic hedgehog signaling direct lung fibroblast precursors during alveolar septal formation. Am J Physiol Lung Cell Mol Physiol 305, L229-239.

Meng, X.M., Tang, P.M., Li, J., and Lan, H.Y. (2015). TGF-beta/Smad signaling in renal fibrosis. Front Physiol 6, 82.

Nieuwenhuis, E., Barnfield, P.C., Makino, S., and Hui, C.C. (2007). Epidermal hyperplasia and expansion of the interfollicular stem cell compartment in mutant mice with a C-terminal truncation of Patched1. Dev Biol 308, 547-560.

Nusslein-Volhard, C., and Wieschaus, E. (1980). Mutations affecting segment number and polarity in Drosophila. Nature 287, 795-801.

O’Hara, W.A., Azar, W.J., Behringer, R.R., Renfree, M.B., and Pask, A.J. (2011). Desert hedgehog is a mammal-specific gene expressed during testicular and ovarian development in a marsupial. BMC Dev Biol 11, 72.

Ochi, H., Pearson, B.J., Chuang, P.T., Hammerschmidt, M., and Westerfield, M. (2006). Hhip regulates zebrafish muscle development by both sequestering Hedgehog and modulating localization of Smoothened. Dev Biol 297, 127-140.

Pan, A., Chang, L., Nguyen, A., and James, A.W. (2013). A review of hedgehog signaling in cranial bone development. Front Physiol 4, 61.

Polizio, A.H., Chinchilla, P., Chen, X., Manning, D.R., and Riobo, N.A. (2011). Sonic Hedgehog activates the GTPases Rac1 and RhoA in a Gli-independent manner through coupling of smoothened to Gi proteins. Sci Signal 4, pt7.

Rangwala, F., Guy, C.D., Lu, J., Suzuki, A., Burchette, J.L., Abdelmalek, M.F., Chen, W., and Diehl, A.M. (2011). Increased production of sonic hedgehog by ballooned hepatocytes. J Pathol 224, 401-410.

Rauhauser, A.A., Ren, C., Lu, D., Li, B., Zhu, J., McEnery, K., Vadnagara, K., Zepeda-Orozco, D., Zhou, X.J., Lin, F., Jetten, A.M., and Attanasio, M. (2015). Hedgehog signaling indirectly affects tubular cell survival after obstructive kidney injury. Am J Physiol Renal Physiol 309, F770-778.

Reichrath, J., and Reichrath, S. (2013). The relevance of the vitamin D endocrine system (VDES) for tumorigenesis, prevention, and treatment 
of non-melanoma skin cancer (NMSC): present concepts and future perspectives. Dermatoendocrinol 5, 38-50.

Robarge, K.D., Brunton, S.A., Castanedo, G.M., Cui, Y., Dina, M.S., Goldsmith, R., Gould, S.E., Guichert, O., Gunzner, J.L., Halladay, J., Jia, W., Khojasteh, C., Koehler, M.F., Kotkow, K., La H., Lalonde, R.L., Lau, K., Lee, L., Marshall, D., Marsters, J.C.Jr., Murray, L.J., Qian, C., Rubin, L.L., Salphati, L., Stanley, M.S., Stibbard, J.H., Sutherlin, D.P., Ubhayaker, S., Wang, S., Wong, S., and Xie, M. (2009). GDC-0449-a potent inhibitor of the hedgehog pathway. Bioorg Med Chem Lett 19, 5576-5581.

Robbins, D.J., Fei, D.L., and Riobo, N.A. (2012). The Hedgehog signal transduction network. Sci Signal 5, re6.

Ruat, M. (2015). The smoothened receptor in cancer and regenerative medicine. In Topics in Medicinal Chemistry (New York: Springer Berlin Heidelberg).

Ruiz-Gomez, A., Molnar, C., Holguin, H., Mayor, F.Jr., and de Celis, J.F. (2007). The cell biology of Smo signalling and its relationships with GPCRs. Biochim Biophys Acta 1768, 901-912.

Santos, P.C., Krieger, J.E., and Pereira, A.C. (2012). Renin-angiotensin system, hypertension, and chronic kidney disease: pharmacogenetic implications. J Pharmacol Sci 120, 77-88.

Strand, M.F., Wilson, S.R., Dembinski, J.L., Holsworth, D.D., Khvat, A., Okun, I., Petersen, D., and Krauss, S. (2011). A novel synthetic smoothened antagonist transiently inhibits pancreatic adenocarcinoma xenografts in a mouse model. PLoS One 6, e19904.

Sweetwyne, M.T., Tao, J., and Susztak, K. (2014). Kick it up a notch: Notch signaling and kidney fibrosis. Kidney Int Suppl 4, 91-96.

Tan, R.J., Zhou, D., Zhou, L., and Liu, Y. (2014). Wnt/beta-catenin signaling and kidney fibrosis. Kidney Int Suppl 4, 84-90.

Teperino, R., Aberger, F., Esterbauer, H., Riobo, N., and Pospisilik, J.A. (2014). Canonical and non-canonical Hedgehog signalling and the control of metabolism. Semin Cell Dev Biol 33, 81-92.

Thibert, C., Teillet, M.A., Lapointe, F., Mazelin, L., Le Douarin, N.M., and Mehlen, P. (2003). Inhibition of neuroepithelial patched-induced apoptosis by sonic hedgehog. Science 301, 843-846.

Tukachinsky, H., Lopez, L.V., and Salic, A. (2010). A mechanism for vertebrate Hedgehog signaling: recruitment to cilia and dissociation of SuFu-Gli protein complexes. J Cell Biol 191, 415-428.

Wang, D., Dai, C., Li, Y., and Liu, Y. (2011). Canonical Wnt/beta-catenin signaling mediates transforming growth factor-beta1-driven podocyte injury and proteinuria. Kidney Int 80, 1159-1169.

Yam, P.T., Langlois, S.D., Morin, S., and Charron, F. (2009). Sonic hedgehog guides axons through a noncanonical, Src-family-kinasedependent signaling pathway. Neuron 62, 349-362.

Yang, L., Wang, Y., Mao, H., Fleig, S., Omenetti, A., Brown, K.D., Sicklick, J.K., Li, Y.X., and Diehl, A.M. (2008). Sonic hedgehog is an autocrine viability factor for myofibroblastic hepatic stellate cells. J Hepatol 48, 98-106.
Yu, J., Carroll, T.J., and McMahon, A.P. (2002). Sonic hedgehog regulates proliferation and differentiation of mesenchymal cells in the mouse metanephric kidney. Development 129, 5301-5312.

Zeisberg, M., and Neilson, E.G. (2010). Mechanisms of tubulointerstitial fibrosis. J Am Soc Nephrol 21, 1819-1834.

Zhang, L., Wang, F., Wang, L., Wang, W., Liu, B., Liu, J., Chen, M., He, Q., Liao, Y., Yu, X., Chen, N., Zhang, J.E., Hu, Z., Liu, F., Hong, D., Ma, L., Liu, H., Zhou, X., Chen, J., Pan, L., Chen, W., Wang, W., Li, X., and Wang, H. (2012). Prevalence of chronic kidney disease in China: a cross-sectional survey. Lancet 379, 815-822.

Zhao, C., Chen, A., Jamieson, C.H., Fereshteh, M., Abrahamsson, A., Blum, J., Kwon, H.Y., Kim, J., Chute, J.P., Rizzieri, D., Munchhof, M., VanArsdale, T., Beachy, P.A., and Reya, T. (2009). Hedgehog signalling is essential for maintenance of cancer stem cells in myeloid leukaemia. Nature 458, 776-779.

Zhao, Q., Beck, A., and Fraidenraich, D. (2010). A direct, non-canonical pathway for Hedgehog proteins in the endothelium. Cell Cycle 9, 647-648.

Zhao, X.P., Liao, M.C., Chang, S.Y., Abdo, S., Aliou, Y., Chenier, I., Ingelfinger, J.R., and Zhang, S.L. (2014). Maternal diabetes modulates kidney formation in murine progeny: the role of hedgehog interacting protein (HHIP). Diabetologia 57, 1986-1996.

Zhou, D., Li, Y., Zhou, L., Tan, R.J., Xiao, L., Liang, M., Hou, F.F., and Liu, Y. (2014). Sonic hedgehog is a novel tubule-derived growth factor for interstitial fibroblasts after kidney injury. J Am Soc Nephrol 25, 2187-2200.

Zhou, D., Li, Y., Lin, L., Zhou, L., Igarashi, P., and Liu, Y. (2012). Tubule-specific ablation of endogenous beta-catenin aggravates acute kidney injury in mice. Kidney Int 82, 537-547.

Zhou, D., and Liu, Y. (2016). Renal fibrosis in 2015: understanding the mechanisms of kidney fibrosis. Nat Rev Nephrol 12, 68-70.

Zhou, D., Tan, R.J., Fu, H., and Liu, Y. (2016). Wnt/beta-catenin signaling in kidney injury and repair: a double-edged sword. Lab Invest 96, 156-167.

Zhou, L., Li, Y., Hao, S., Zhou, D., Tan, R.J., Nie, J., Hou, F.F., Kahn, M., and Liu, Y. (2015). Multiple genes of the renin-angiotensin system are novel targets of Wnt/beta-catenin signaling. J Am Soc Nephrol 26, $107-120$.

Zhou, L., and Liu, Y. (2015). Wnt/beta-catenin signalling and podocyte dysfunction in proteinuric kidney disease. Nat Rev Nephrol 11, 535-545.

Zhulyn, O., Nieuwenhuis, E., Liu, Y.C., Angers, S., and Hui, C.C. (2015). Ptch2 shares overlapping functions with Ptch1 in Smo regulation and limb development. Dev Biol 397, 191-202.

Zoccali, C., Kramer, A., and Jager, K.J. (2010). Epidemiology of CKD in Europe: an uncertain scenario. Nephrol Dial Transplant 25, 1731-1733.

Open Access This article is distributed under the terms of the Creative Commons Attribution License which permits any use, distribution, and reproduction in any medium, provided the original author(s) and source are credited. 